\title{
Reducing Transaction Abort Rate of Epidemic Algorithm in Replicated Databases
}

\author{
Huaizhong Lin ${ }^{1, *}$, Zengwei Zheng ${ }^{1,2}$, and Chun Chen ${ }^{1}$ \\ ${ }^{1}$ College of Computer Science, Zhejiang University, 310027 Hangzhou, China \\ \{linhz, zhengzw, chenc\}@zju.edu.cn \\ ${ }^{2}$ City College, Zhejiang University, 310015 Hangzhou, China \\ zhengzwazucc. edu.cn
}

\begin{abstract}
Easy to deploy, robust, and highly resilient to failures, epidemic algorithms are a potentially effective mechanism for propagating information in large peer-to-peer systems deployed on Internet or ad hoc networks. In the paper, we explore the epidemic algorithms used for transaction processing in replicated databases that reside in weakly connected environments. We concentrate on the transaction commit voting process of the epidemic algorithms and suggest a new voting method, which takes an optimistic approach in conflict reconciliation. The optimistic voting protocol decreases abort rate and improves average response time of transactions.
\end{abstract}

\section{Introduction}

In recent years, the wireless communication and wide area network technologies, especially Internet, evolve rapidly. Weakly connected environments, which are characterized by low bandwidth, excessive latency, instability of connection, and constant disconnection, are used more and more frequently. Data replication is the common approach to improve system performance and availability. But due to the massive communication overhead in weakly connected environments, eager replication may bring about unacceptable number of failed or blocked transactions, and result in dramatic drop of system performance $[1,2]$.

Epidemic algorithms [3], which mimic the spread of a contagious disease, have recently gained popularity as a potentially effective solution for disseminating information in large-scale systems, particularly P2P systems deployed on Internet or ad hoc networks. In addition to their inherent scalability, they are easy to deploy, robust, and resilient to failure. It is possible to adjust the parameters of an epidemic algorithm to achieve high reliability despite process crashes and disconnections, packet losses, and a dynamic network topology.

Epidemic algorithms can be used for managing replicated data [4-9]. In an epidemic approach, sites perform update operations locally and communicate peer-topeer in a lazy manner to propagate updates. Transactional consistency is achieved by

* Supported by the Natural Science Fundation of Zhejiang Province, China (Grant no. M603230) and the Research Fund for Doctoral Program of Higher Education from Ministry of Education, China (Grant no. 20020335020). 
decentralized conflict detect and reconciliation. Sites communicate in a way that maintains the causal order of updates and the communication can pass through one or more intermediate sites. Therefore, the epidemic model provides an environment that is tolerant of communication failures and doesn't require continuous connection between sites. Epidemic model is suitable for transaction processing of replication systems in weakly connected environments.

Several protocols have been proposed for implementing epidemic model in replicated databases, like ROWA (Read-One Write-All) protocol [4], quorum protocol [5], voting protocol $[7,8]$, etc. In this paper, we describe the optimistic voting protocol, which introduces condition and order vote in the election process in transaction commitment. Condition vote postpones the final decisions on conflicting transactions and therefore improves the chances for transactions to get yes vote. Order vote prescribes the commit order of transactions that have read-write and write-write conflicts and eliminates transaction aborts due to these kinds of data conflicts. Optimistic voting protocol reduces abort rate and improves average response time of transactions when compared to other protocols.

The rest of the paper is organized as follows. In section 2, we develop the necessary background and introduce the epidemic model used in replicated databases. In section 3, we describe the optimistic voting protocol. In section 4, we perform the performance evaluation. We conclude the paper in section 5.

\section{Epidemic Model}

We consider a distributed system consisting of $n$ sites labeled $S_{1}, S_{2}, \ldots, S_{n}$ and data items replicated fully or partially at all sites. Epidemic model assumes a fail-stop model of site failures and an unreliable communication medium. Sites communicate each other through messages passing in a pair-wise manner. Messages can arrive in any order, take an unbounded amount of time to arrive, or may be lost entirely, however, messages will not arrive corrupted. For this reason, timeout is not used in the protocols to detect conflicts and deadlocks.

Epidemic model is based on the causal delivery of log records where each record corresponds to one transaction instead of one operation. An event model [5] is used to describe the system execution, $(\mathrm{E}, \rightarrow)$, where $\mathrm{E}$ is a set of transaction events and $\rightarrow$ is the happened-before relation which is a partial order on all events in E. The partial order $\rightarrow$ satisfies the following two conditions:

(1) Events occurring at the same site are totally ordered;

(2) If $\mathrm{e}$ is a sending event and $\mathrm{f}$ is the corresponding receiving event, then $\mathrm{e} \rightarrow \mathrm{f}$.

Vector clocks are used to ensure the property that if two events are causally ordered, their effects should be applied in that order at all sites. Each site $S_{k}$ keeps a two-dimension time-table, which corresponds to $S_{k}$ 's most recent knowledge of the vector clocks at all sites. Upon communication, $S_{k}$ sends a message including its own time-table and all records that receiving site hasn't received. Then the receiving site processes the events according to causal order and incorporates the time-table in an atomic step to reflect the new information from $S_{k}$. 
The site $S_{k}$ determines the records that receiving site $S_{j}$ hasn't received according the following predicate [5]:

$\operatorname{HasRecvd}\left(\mathrm{T}_{\mathrm{k}}, \mathrm{t}, \mathrm{S}_{\mathrm{j}}\right) \equiv \mathrm{T}_{\mathrm{k}}[\mathrm{j}$, Site $(\mathrm{t})] \geq \operatorname{Time}(\mathrm{t})$

Where $t$ is an event, Site $(t)$ is the site at which $t$ occurred, and Time(t) is the local time at Site(t) when t occurred.

Upon completion of operations, a read-only transaction can be committed locally whereas an update transaction pre-commits and becomes a candidate. The read set, write set, and the update values of the candidate are recorded in log. Then sites exchange their respective log records to detect global conflicts and propagate values written by the transaction. A candidate is voted on and is eventually either committed (if it wins a plurality of the total system votes) or aborted.

When a transaction pre-commits, it is attached with a global distinct timestamp denoted by (local_ts, site_index), which is composed of a local timestamp and a distinct site index. Formally, we define a total order $<$ on timestamps as follows. Suppose two timestamps $\operatorname{ts}\left(\mathrm{T}_{1}\right)=\left(\right.$ local_ts $_{1}$, site_index $\left.{ }_{1}\right)$ and $\mathrm{ts}\left(\mathrm{T}_{2}\right)=\left(\right.$ local_ts $_{2}$, site_index $\left.{ }_{2}\right)$, then $\operatorname{ts}\left(\mathrm{T}_{1}\right)<\mathrm{ts}\left(\mathrm{T}_{2}\right)$ if and only if:

(1) local_ts $<$ local_ts 2 , or

(2) local_ts ${ }_{1}=$ local_ts $_{2}$ and site_index $x_{1}<$ site_index 2 .

The information of local timestamp is piggybacked in the usual epidemic messages and a site adjusts its local timestamp as follows [10]: when site A receives a message from site $\mathrm{B}$, it advances its local timestamp to $\max \left\{\right.$ local_ts ${ }_{\mathrm{A}}$, the local_ts $\mathrm{B}_{\mathrm{B}}$ carried by message $\}$. If there are no communications between sites, their local timestamps will drift apart. But this doesn't matter since, in the absence of such communications, there is no need for synchronization in the first place and the drift will not affect the correctness of the protocol.

\section{Optimistic Voting Protocol}

\subsection{Condition and Order Vote}

Suppose two conflicting transactions $T_{i}$ and $T_{j}$ are issued by two sites concurrently. To maintain serializability, previous epidemic protocols consider that there is only one transaction can be committed and each site can only cast yes vote to one transaction in election, for example $\mathrm{T}_{\mathrm{i}}$. In optimistic voting protocol, to increase the chances to get yes vote for transaction $\mathrm{T}_{\mathrm{j}}$, sites can cast condition vote on it (whereas it is cast no vote in quorum or voting protocols). The condition vote on $\mathrm{T}_{\mathrm{j}}$ can be transformed to yes vote if $\mathrm{T}_{\mathrm{i}}$ is aborted. The use of condition vote postpones the final vote decision on transactions.

Definition 1. When voting on transaction $T$, suppose $C=\left\{T_{1}, \ldots, T_{p}\right\}$ is the set in which each transaction conflicts with $\mathrm{T}$, the condition vote $\operatorname{cond}(\mathrm{C})$ means that it can be transformed to yes vote in case each transaction in $\mathrm{C}$ is aborted, otherwise to no vote.

The transform rules of condition vote are as follows:

(1) If $\exists \mathrm{T}_{\mathrm{i}} \in \mathrm{C}, \mathrm{T}_{\mathrm{i}}$ has been aborted, then $\operatorname{cond}(\mathrm{C}) \rightarrow \operatorname{cond}\left(\mathrm{C}-\mathrm{T}_{\mathrm{i}}\right)$;

(2) If $\forall \mathrm{T}_{\mathrm{i}} \in \mathrm{C}, \mathrm{T}_{\mathrm{i}}$ has been aborted, then $\operatorname{cond}(\mathrm{C}) \rightarrow$ yes;

(3) If $\exists \mathrm{T}_{\mathrm{i}} \in \mathrm{C}, \mathrm{T}_{\mathrm{i}}$ has been committed, then $\operatorname{cond}(\mathrm{C}) \rightarrow$ no. 
For two transactions $\mathrm{T}_{\mathrm{i}}$ and $\mathrm{T}_{\mathrm{j}}$ that only have read-write and write-write conflicts, if the correct order can be preserved at all sites, e.g. $T_{i}$ is committed before $T_{j}$, then the two conflicting transactions $\mathrm{T}_{\mathrm{i}}$ and $\mathrm{T}_{\mathrm{j}}$ can all be committed maintaining consistency. Order vote prescribes the commit order of these kinds of conflicting transactions. Additionally, it is easily observed that condition and order vote can coexist on one transaction $\mathrm{T}$.

Definition 2. When voting on transaction $T$, suppose $C=\left\{T_{1}, \ldots, T_{p}\right\}$ is the set in which each transaction has only read-write and write-write conflicts with $\mathrm{T}$, the order vote $\operatorname{order}(\mathrm{C})$ means that it can be transformed to yes vote when all transactions in $\mathrm{C}$ have been committed or aborted at one site.

The transform rule of order vote is as follows:

If at one site, $\forall \mathrm{T}_{\mathrm{i}} \in \mathrm{C}, \mathrm{T}_{\mathrm{i}}$ has been committed or aborted, then $\operatorname{order}(\mathrm{C}) \rightarrow$ yes .

Each site $S_{k}$ maintains a list of candidates by the receiving order. Let list $t_{k}$ denote the candidate set in which the vote on each transaction by the site is not no vote. When $S_{k}$ receives a new candidate $T$, it votes on $T$ according to the following rules. For convenience of description, Let

cond_set $=\left\{\mathrm{T}_{\mathrm{i}} \mid \mathrm{T}_{\mathrm{i}} \in\right.$ list $_{\mathrm{k}}$, wr_conflict $\left(\mathrm{T}_{\mathrm{i}}, \mathrm{T}\right)$ is true $\}$,

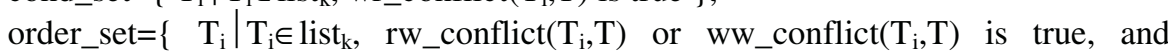
wr_conflict $\left(\mathrm{T}_{\mathrm{i}}, \mathrm{T}\right)$ is false $\}$.

(1) If $\exists x \in \operatorname{ReadSet}(T), \operatorname{ReadVN}(T, x)<\operatorname{CurrVN}\left(S_{k}, x\right)$, it means that the value read by $\mathrm{T}$ has been overwritten, then vote no;

(2) If cond_set $=\varnothing$ and order_set $=\varnothing$, it means that there are no transactions in list $_{\mathrm{k}}$ that have conflict with $\mathrm{T}$, then vote yes;

(3) If $\exists \mathrm{T}_{\mathrm{i}} \in \mathrm{cond} \_$set $\cup$ order_set, ts $\left(\mathrm{T}_{\mathrm{i}}\right)>\mathrm{ts}(\mathrm{T})$, then vote $n o$;

(4) If $\forall \mathrm{T}_{\mathrm{i}} \in \mathrm{cond} \_s e t \cup o r d e r \_s e t, t \mathrm{ts}\left(\mathrm{T}_{\mathrm{i}}\right)<\mathrm{ts}(\mathrm{T})$, then vote cond(cond_set) + or der(order_set). The '+' denotes that the vote is transformed to yes vote if and only if both the condition and order vote are transformed to yes, otherwise to no vote.

The correctness proof of optimistic voting can be found in [9].

The votes collected in optimistic voting protocol can be viewed as optimistic quorum. The optimistic quorum differs from ordinary quorum in replicated databases in that the quorum is conditional and can only be transformed to really quorum based on the results of other transactions. This optimistic quorum increases the chance for a transaction to win a majority of sites, thus reducing the transaction abort rate.

\subsection{An Example}

We explain the optimistic voting protocol with an example. Suppose three transactions $\mathrm{T}_{1}, \mathrm{~T}_{2}$ and $\mathrm{T}_{3}\left(\mathrm{ts}\left(\mathrm{T}_{1}\right)<\mathrm{ts}\left(\mathrm{T}_{2}\right)<\mathrm{ts}\left(\mathrm{T}_{3}\right)\right)$.

Fig. 1 shows the voting process of voting protocol. Because three transactions have data conflict with each other, only one transaction can be committed. Fig. 2 shows optimistic voting process, which avoid the abort of $\mathrm{T}_{3}$ by use of order vote. From the figures, we observe that $\mathrm{T}_{2}$ in optimistic voting can be committed earlier than in 
voting protocol by use of order vote. For clarity, we omit some unimportant information exchanges in Fig. 1 and Fig. 2.

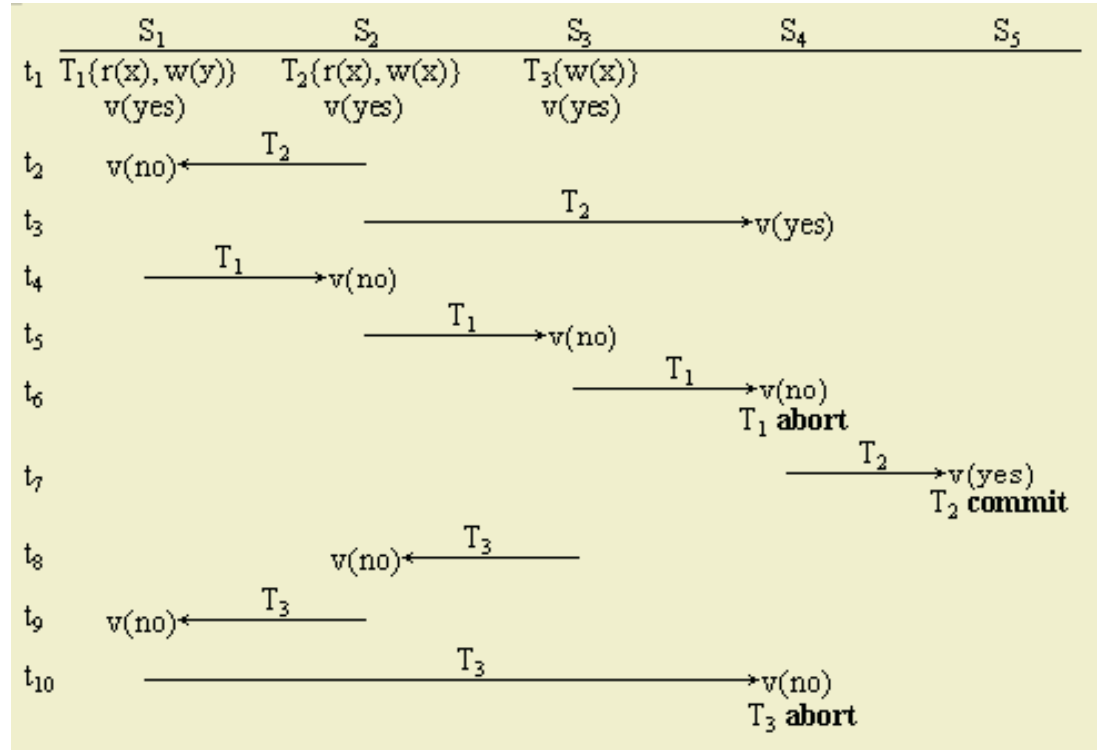

Fig. 1. Voting protocol

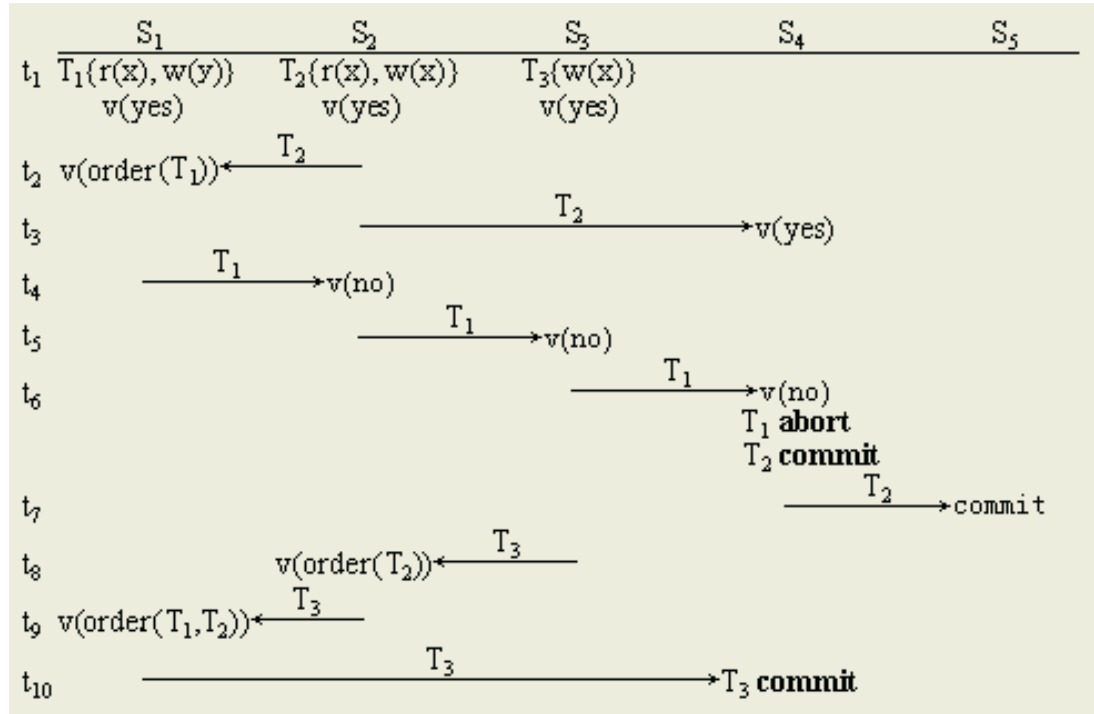

Fig. 2. Optimistic voting protocol 


\section{Performance Evaluation}

We perform experiments to show performance improvement attained by optimistic voting $(\mathrm{OV})$ protocol. Additionally, we investigate two representative epidemic replication schemes from the literature, ROWA protocol [4] and voting protocol [7] (quorum protocol [5] is similar to voting protocol). The evaluations are done at 10 desktops connected via a 10Mbps Ethernet network.

The simulation assumes that data items are fully replicated at all sites and tickets are uniformly distributed among sites. Each site generates transactions randomly according to a global transaction generation rate. Data items are accessed uniformly by transactions. Each site periodically initiates a synchronization session with a given synchronization interval by sending a pull request to another randomly selected site.

Since we focus on the transaction abort rate and commit delay of different protocols, we don't model any read-only transactions. Each transaction read 5-10 data items and write 5 data items that are in the read set, so there are no blind writes. The main parameters and settings used in the experiments are summarized in Table 1.

Table 1. Experimental parameters

\begin{tabular}{lll}
\hline Parameters & Descriptions & Values \\
\hline $\mathrm{N}$ & Site number & 10 \\
Sync. interval & Average synchronization interval & $1 \sim 5 \mathrm{~s}$ \\
Trans. rate & Average generation rate of update transactions & $0.2 \sim 20 / \mathrm{s}$ \\
Data items & Total data item number & 500 \\
\hline
\end{tabular}

Fig.3 illustrates the transaction abort rate of three protocols for various values of transaction generation rate. From the figures, it is obvious that optimistic voting protocol outperforms the other two protocols.

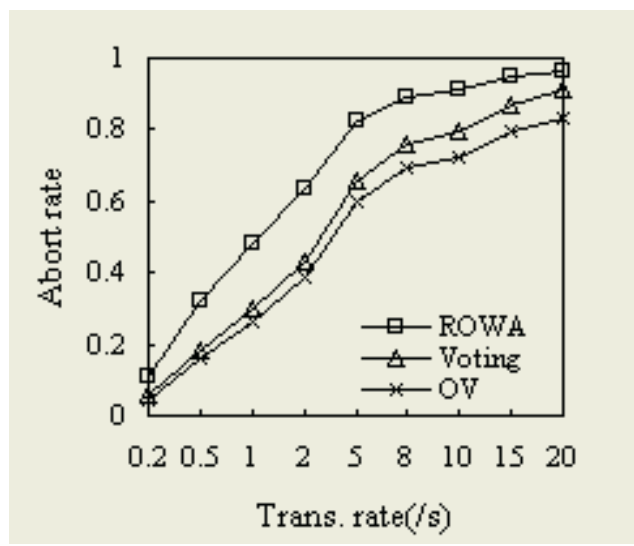

Fig. 3. Abort rate vs. transaction generation rate (Synchronization interval=1.0s) 
In optimistic voting protocol presented above, the condition and order vote of a transaction is dependent on other transactions. This dependency relation in optimistic voting is one-way, i.e. a transaction can only depend on transactions that have smaller global timestamp than it (noted as protocol A). The one-way dependency ensures that there are no cycles among transactions and therefore no global deadlocks. This oneway dependency can be converted to depending on transactions that have larger global timestamp (noted as protocol B). We explore the impacts on performance of different dependency direction by experiments. Fig. 4 and Fig.5 illustrate the transaction abort rate of protocol $\mathrm{A}$, protocol $\mathrm{B}$, and voting protocol for various values of transaction generation rate and synchronization interval. From the figures, it is obvious that protocol $\mathrm{A}$ is better than protocol $\mathrm{B}$ with average $5.4 \%$ performance gain. It is the natural direction for a transaction to depend on other transactions with smaller global timestamp. The transaction with smaller timestamp has been stay in the system for a longer time span and will be committed or rollbacked much earlier, which makes the transform of condition and order vote more quickly, thus reduce the delay of a transaction in the system. Additionally, we can notice that both protocol A and B outperforms the voting protocol.

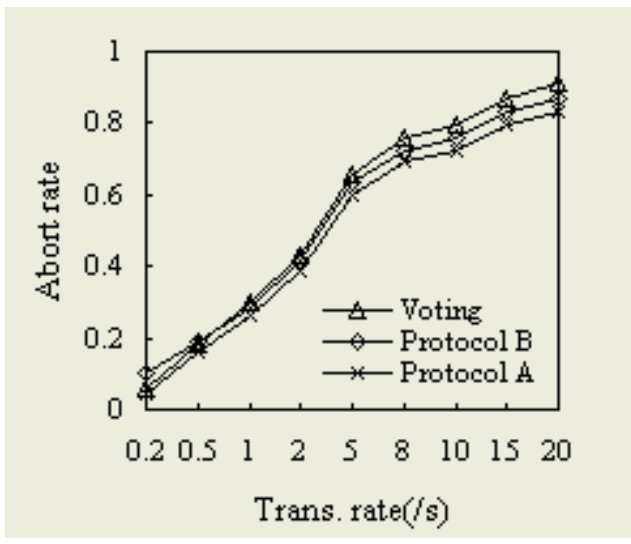

Fig. 4. Abort rate vs. transaction generation rate (Synchronization interval=1.0s)

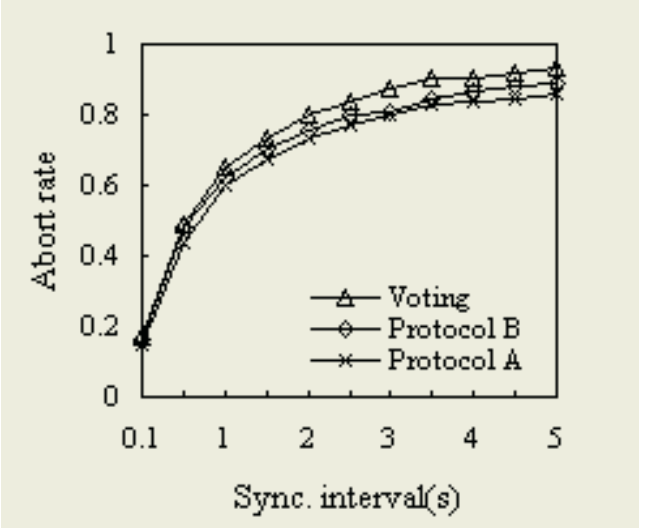

Fig. 5. Abort rate vs. synchronization interval (Transaction generation rate=5.0/s) 


\section{Conclusion}

Epidemic replication schemes are used extensively in transaction processing in weakly connected environments. Some continuously connected systems also use epidemic model to improve system efficiency. The optimistic voting protocol presented in this paper improves system performance in epidemic model and is of high practical values.

\section{References}

1. J. Gray, P. Helland, P. O'Neil, and D. Shasha. The dangers of replication and a solution. In Proceedings of ACM SIGMOD International Conference on Management of Data. Montreal, Canada, 1996. 173-182.

2. T. Anderson, Y. Breitbart, H. F. Korth, and A. Wool. Replication, consistency, and practicality: are these mutually exclusive. In Proceedings of ACM SIGMOD International Conference on the Management of Data. Seattle, Washington, 1998. 484-495.

3. Patrick T. Eugster, Rachid Guerraoui, Anne-Marie Kermarrec, and Laurent Massoulié. Epidemic Information Dissemination in Distributed Systems. IEEE Computer, 2004, (5): 60-67.

4. D. Agrawal, A. El Abbadi, and R. Steinke. Epidemic algorithms in replicated databases. In Proceedings of 16th ACM SIGACT-SIGMOD-SIGART Symposium on Principles of Database Systems. Tucson, Arizona, 1997. 161-172.

5. J. Holliday, R. Steinke, D. Agrawal, and A. El Abbadi. Epidemic quorums for managing replicated data. In Proceedings of 19th IEEE International Performance, Computing, and Communications Conference. Phoenix, Arizona, 2000. 93-100.

6. K. Petersen, M. J. Spreitzer, D. B. Terry, M. M. Theimer, and A. J. Demers. Flexible update propagation for weakly consistent replication. In Proceedings of 16th ACM Symposium on Operating System Principles. St. Malo, France, 1997. 288-301.

7. U. Çetintemel, P. J. Keleher, and M. J. Franklin. Support for speculative update propagation and mobility in Deno. In Proceedings of 21st International Conference on Distributed Computing Systems. Phoenix, Arizona: IEEE Computer Society Press, 2001. 509-516.

8. P. J. Keleher. Decentralized replicated object protocols. In Proceedings of 18th Annual ACM Symposium on Principles of Distributed Computing. Atlanta, Georgia, 1999. 143-151.

9. Huaizhong Lin and Chun Chen. Optimistic voting for managing replicated data. Journal of Computer Science and Technology, 2002, 17(6): 874-881.

10. M. M. Deris, A. Mamat, and M. P. Hamzah. Replicated data management for transactions sharing in distributed database. In Proceedings of 4th International Conference/Exhibition on High Performance Computing in Asia-Pacific Region. Beijing, China: IEEE Computer Society Press, 2000. 836-841. 